\title{
Application of the Gray Wolf (GWO) algorithm in the tuning of a PID controller in a feedback control system
}

\author{
Antonio B.L. de Medeiros ${ }^{1}$ \\ ${ }^{1}$ Universidade Federal do Pará. Rua Augusto Corrêa, 01 - Guamá. CEP 66075-110 - Belém Pará - Brasil.PA \\ Email: benjaminleao@gmail.com \\ Received: November $24^{\text {th }}, 2017$. \\ ABSTRACT \\ Accepted:. November 30 ${ }^{\text {th }}, 2017$. \\ In this paper, a PID controller will have its parameters optimized by the Gray Wolf (GWO) \\ algorithm. This will determine the values of $\mathrm{K}$ and a of the controller in order to guarantee, in a \\ Published:. December $13^{\text {th }}, 2017$. \\ response to the unitary step, an overshooting of $10 \%$. The performance of the algorithm will be \\ evaluated by the error between the desired value of the overshooting and the one found after the \\ iterations. A comparative study will be made between the GWO optimization and the classic trial \\ Copyright (02016 by authors and Institute \\ of Technology Galileo of Amazon (ITEGAM). \\ This work is licensed under the Creative \\ and error method (T\&E) adopted in the literature.
} Commons Attribution International License (CC BY 4.0).

http://creativecommons.org/licenses/by/4.0/

Keywords: Optimization, PID, parameters, performance, GWO.

\section{Aplicação do algoritmo do lobo cinzento (GWO) na sintonia de um controlador PID em um sistema de controle retroalimentado}

\section{RESUMO}

Neste artigo, um controlador PID terá seus parâmetros otimizados pelo algoritmo do lobo cinzento (GWO). Este, irá determinar os valos de $K$ e $a$ do controlador a fim de garantir, a uma resposta ao degrau unitário, um sobressinal máximo de 10\%. O desempenho do algoritmo será avaliado pelo erro entre o valor desejado do sobressinal e o encontrado após as iterações. Será feito um estudo comparativo entre a otimização pelo GWO e o método clássico de tentativa e erro (T\&E) adotado na literatura.

Palavras-chave: Otimização, PID, parâmetros, desempenho, GWO.

\section{INTRODUÇÃO}

O controle automático é essencial em qualquer campo da engenharia e da ciência e é um componente importante e intrínseco em sistemas de veículos espaciais, sistemas robóticos, modernos sistemas de manufatura e quaisquer operações industriais que envolvam o controle de temperatura, pressão, viscosidade, umidade, vazão, etc. [1]. Os controladores PID aparecem como a alternativa mais utilizada nos ramos da indústria. Apesar da sua popularidade, os controladores PID são, muitas vezes, sintonizados manualmente por meio do procedimento de tentativa e de erro. Isso, de certa forma, torna o ajuste do processo difícil e ineficiente [2]. Vários métodos de otimização computacional auxiliam a determinação dos parâmetros destes controladores, e um desses métodos é o GWO (do inglês Grey Wolf Optmizer) criado por [3]. Este trabalho apresenta uma proposta de otimização para a sintonia de um controlador PID utilizando o GWO para a escolha otimizada dos seus parâmetros aplicado a um sistema de controle retroalimentado.

\section{REVISÃO BIBLIOGRÁFICA}

\section{II.1 CONTROLADORES PID}

Os controladores PID são os mais comumente utilizados em aplicações industriais. $\mathrm{O}$ fator decisivo que determina seu uso é o número de parâmetros (ganho proporcional, tempo integrativo e tempo derivativo) e as regras simples que definem seus valores [4]. Este controlador é a agregação de três unidades de sub controle: a proporcional, a integral e a derivativa. O modo de controle proporcional modifica o sinal de controle numa taxa proporcional ao sinal de erro $(e)$ e ao parâmetro proporcional chamado de ganho proporcional $\left(K_{p}\right)$. A parte integral produz uma mudança corretiva na saída, levando o erro o mais próximo de 0 e seu parâmetro ajustável é chamado de tempo integrativo $\left(T_{i}\right)$. O Controle 
derivativo faz com que o loop de controle responda de forma mais rápida, com o menor sobressinal possível, determinado pelo parâmetro tempo derivativo $\left(T_{d}\right)$ [5]. O sinal de controle $u(\mathrm{t})$ gerado por um controlador PID é dado na eq. 2.1:

$$
\begin{gathered}
u(t)=K_{p} e(t)+K_{i} \int_{0}^{t} e(t) d t+K_{d} \frac{d e(t)}{d t} \\
K_{i}=\frac{K_{p}}{T_{i}} \\
K_{d}=K_{p} T_{d}
\end{gathered}
$$

Onde:

$K_{i}$ : Ganho integrativo.

$K_{d}$ : Ganho derivativo.

Aplicando a transformada de Laplace na eq. 2.1, tem-se que:

$$
C(s)=K_{p}+\frac{K_{i}}{s}+K_{d} s
$$

Desenvolvendo a eq. 2.4 nos termos das eq. 2.2 e 2.3 , e adotando que os polos do controlador serão complexos e conjugados, a eq. 2.4 pode ser reescrita na forma da eq. 2.5 :

$$
C(s)=K \frac{(s+a)^{2}}{s}
$$

Onde $K$ e $a$ são constantes.

\section{2 ALGORITMO DO LOBO CINZENTO (GWO)}

O algoritmo do lobo cinzento (GWO - do inglês Grey Wolf Optimizer) é uma técnica de otimização computacional criada por [3] baseada no comportamento de caça dos lobos cinzentos (Canis lupus). Esta espécie vive geralmente em alcateias de 5 a 12 indivíduos. Estes adotam uma hierarquia bem definida e restrita. $\mathrm{O}$ líder dos lobos é chamado de a Alpha $(\alpha)$, que é responsável por tomar decisões relacionadas a caça, tempo, lugar de descanso, etc.

O segundo nível é chamado de Beta $(\beta)$, que dá suporte para os Alphas tomarem as decisões. Estes também são os fortes candidatos a tomarem o lugar de liderança na perda de um Alpha. O nível mais baixo da hierarquia é ocupado pelos Ômegas $(\omega)$, que fazem o papel de bode expiatório e devem satisfazer todo o grupo. O terceiro nível é ocupado pelo Delta $(\delta)$, responsável pela segurança da alcateia [6]. A figura 1 representa a estrutura hierárquica desta espécie.

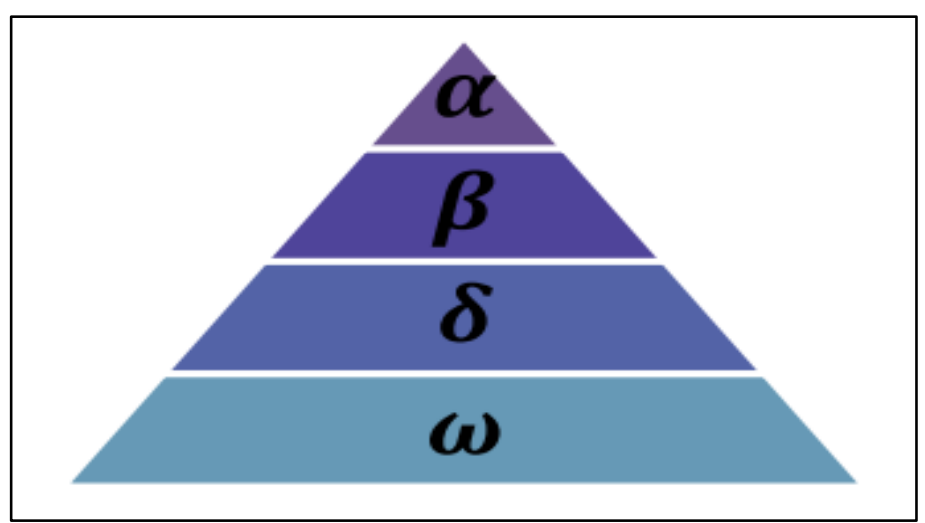

Figura 1: Hierarquia dos lobos cinzentos.

Fonte: [3].

O comportamento de caça é divido em duas etapas fundamentais [7]:

1. Caçar e formar um cerco na presa.
2. Atacar a presa.

A hierarquia social dos lobos, discutida acima, é baseada principalmente no nível de sua dominância. A aptidão dos lobos também depende do seu comportamento dominante, pois o lobo com maior aptidão vai apresentar melhor desempenho e capacidade de tomar decisões durante o processo de caça. Logo, o lobo que está no primeiro nível de hierarquia $(\alpha)$ é considerado o mais apto entre eles [8]. O processo de caça é feito em quatro comportamentos específicos, modelados matematicamente como se segue:

A. Cercando a presa formulado:

Durante o processo de caça, os lobos cercam a presa, assim

$$
\begin{aligned}
& \vec{D}=|\vec{C} \cdot \vec{X} p(t)-\vec{X}(t)| \\
& \vec{X}(t+1)=\vec{X} p(t)-\vec{A} \cdot \vec{D}
\end{aligned}
$$

Onde: $t$ é a iteração atual, $\vec{A}$ e $\vec{C}$ são coeficientes vetoriais, $\vec{X} p$ é o vetor de posição da presa e $\vec{X}$ é o vetor de posição de um lobo cinzento.

Os vetores $\vec{A}$ e $\vec{C}$ são calculados como se segue:

$$
\begin{aligned}
& \vec{A}=2 \vec{a} \cdot \vec{r} 1-\vec{a} \\
& \vec{C}=2 \cdot \vec{r} 2
\end{aligned}
$$

Onde $a$ decai de 2 até 0 no decorrer das iterações e $r 1$ e $r 2$ são vetores de valores randômicos no intervalo [0 1].

\section{B. Caçando a presa}

O comportamento dos lobos cinzentos consiste em reconhecer o local da presa e cercá-la. Contudo, não há como ter a certeza do local exato dela. Para simular este comportamento, é suposto que os alfas, betas e deltas têm o melhor conhecimento do sobre o possível local da presa. Então, os três melhores são escolhidos, e os demais são forçados a segui-los. A modelagem deste comportamento é proposta abaixo:

$$
\vec{D} \alpha=|\vec{C} 1 \cdot \vec{X} \alpha-\vec{X}|, \vec{D} \beta=|\vec{C} 2 \cdot \vec{X} \beta-\vec{X}|, \vec{D} \delta=|\vec{C} 3 \cdot \vec{X} \delta-\vec{X}|
$$

$$
\vec{X} 1=\vec{X} \alpha-\vec{A} 1 \cdot(\vec{D} \alpha), \vec{X} 2=\vec{X} \beta-\vec{A} 2 \cdot(\vec{D} \beta), \vec{X} 1=\vec{X} \alpha-\vec{A} 1 \cdot
$$

$(\vec{D} \alpha)$

$$
\vec{X}(t+1)=\frac{\vec{X} 1+\vec{X} 2+\vec{X} 3}{3}
$$

\section{Atacando a presa}

Os lobos cinzentos, primeiramente, encurralam a presa e quando ela perde sua energia e para de se mover, o líder mais próximo dela a ataca. O Coeficiente do vetor $A$ é decrementado para reduzir a distância entre a posição da presa e a posição dos lobos. Para reduzir o valor de $A$, o valor de $a$ deve ser minimizado como se segue [8]:

$$
a=2-\left(\frac{2}{\text { max.iter }}\right)
$$

Onde max. iter é o número máximo de iterações adotadas na simulação e $a$ é decrescido de 2 a 0 . A figura 2 apresenta os deslocamentos dos lobos nos espaços bidimensional e tridimensional. 


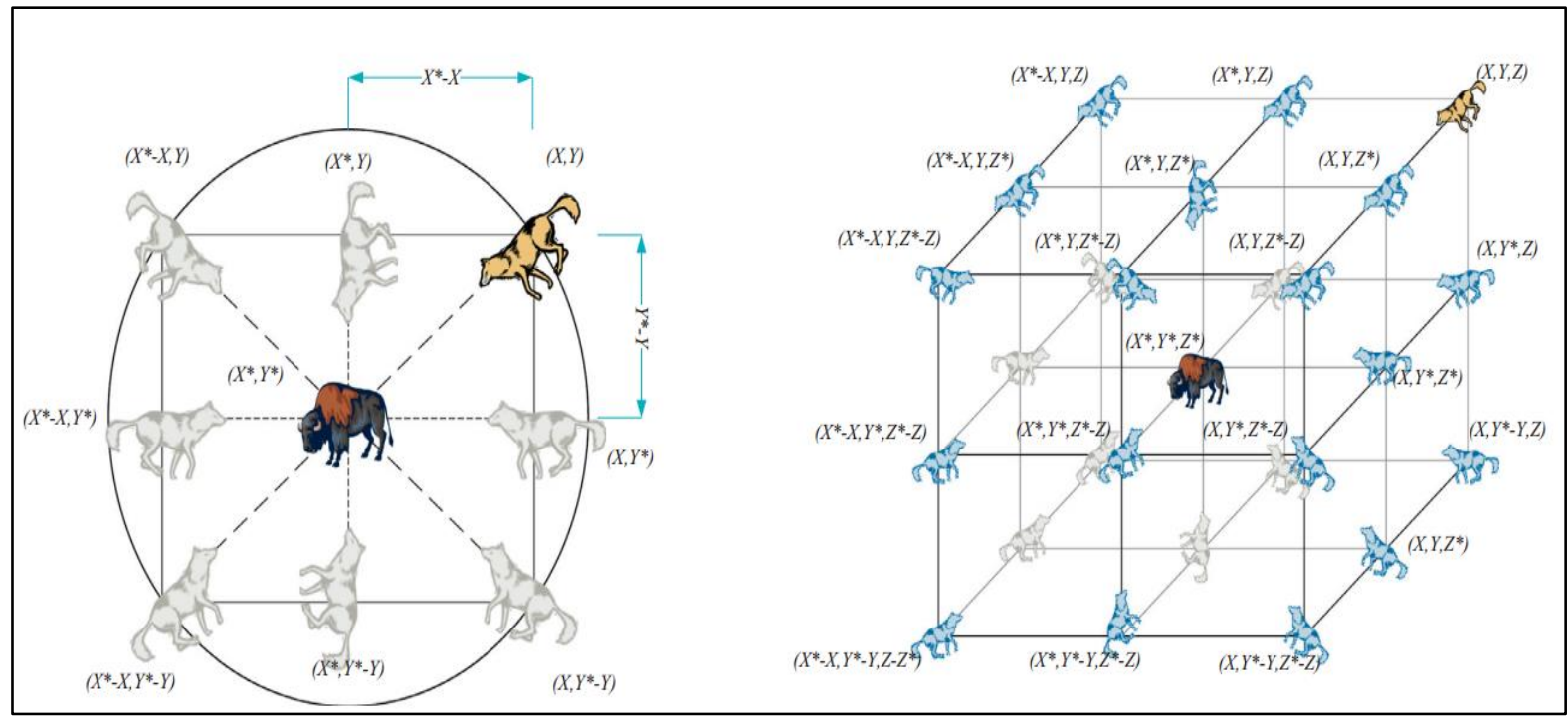

(a)

Figura 2: Deslocamento dos lobos. (a) 2D. (b) 3D.

Fonte: [3]

A figura 3 apresenta o fluxograma do algoritmo do lobo cinzento (GWO):

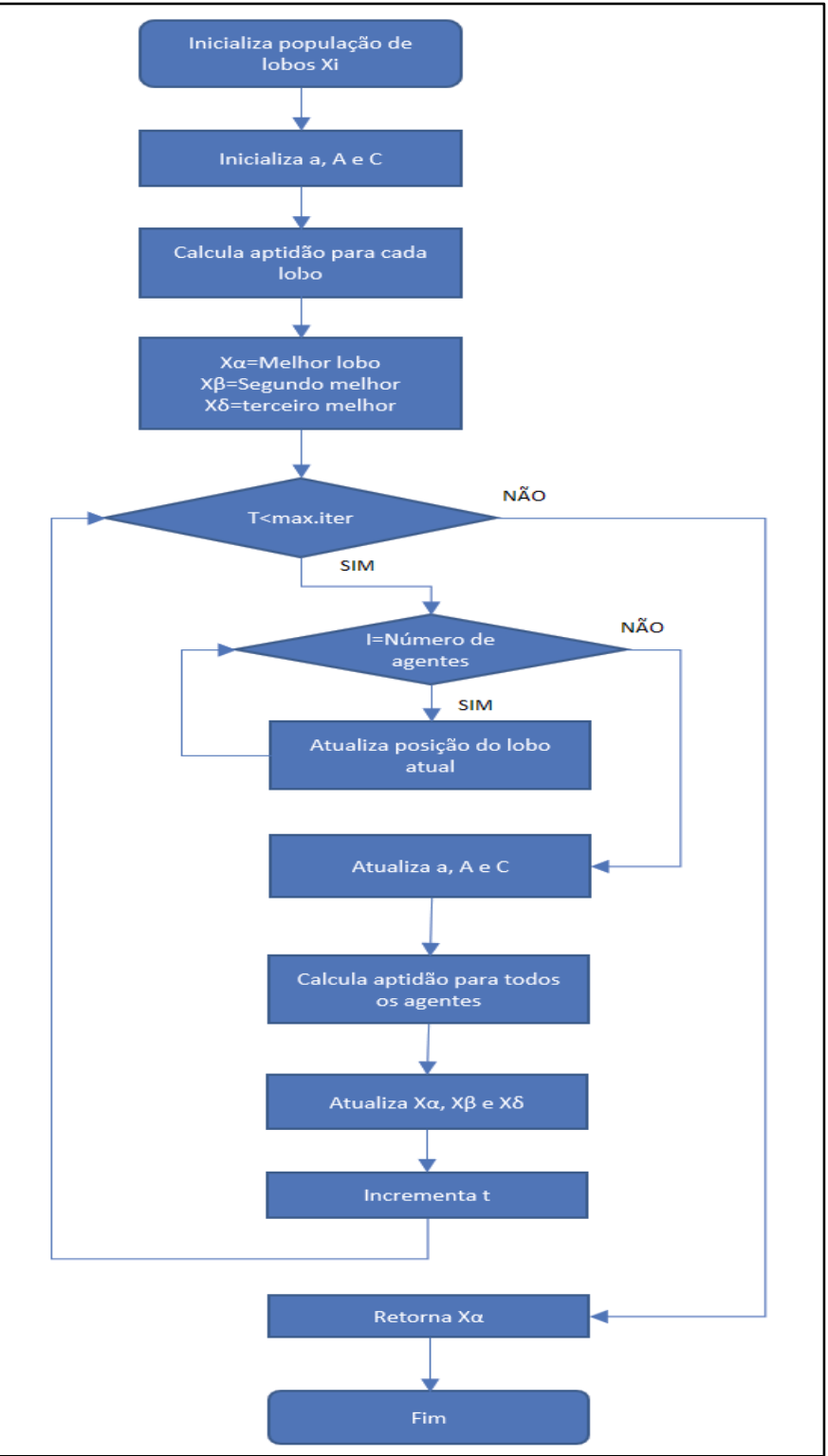

Figura 3: Fluxograma do algoritmo do lobo cinzento (GWO). Fonte: Autor, 2017.

\section{MATERIAIS E MÉTODOS}

\section{III.1 SISTEMA DE CONTROLE}

O problema a seguir foi definido por [1], que utilizou o método de tentativa e erro para sua resolução. Será feita, primeiramente, a otimização com o GWO. Para o sistema de controle dado pela figura 4, deseja-se encontrar uma combinação dos valores de $K$ e $a$ para que o sistema de controle de malha fechada seja subamortecido e com sobressinal de $10 \%$.

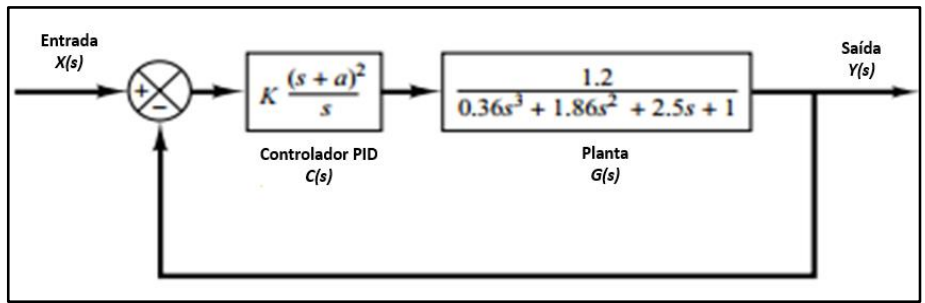

Figura 4 - Sistema de controle de malha fechada.

Fonte: [1]

O primeiro ponto a ser definido é o intervalo de busca destes parâmetros. Os mesmos não podem ser escolhidos de forma exorbitante a ponto de trazer o sistema a instabilidade. Serão utilizados os intervalos definidos pelo autor, supondo que estes mantêm o sistema estável. Logo, tem-se que:

$$
\begin{aligned}
& 2 \leq K \leq 3 \\
& 0,5 \leq a \leq 1,5
\end{aligned}
$$

Aplica-se então um sinal degrau ao sistema em malha fechada, definido pela função de transferência simplificada do sistema de controle.

$$
\begin{aligned}
& G(s)=\frac{1,2}{0,36 s^{3}+1,86 s^{2}+2,5 s+1} \\
& H(s)=1 \\
& X(s)=1
\end{aligned}
$$

Aplicando a retroalimentação do sistema e combinando as eq. $5,16,17$ e 18 , resulta a eq. 19

$$
Y(s)=X(s) \frac{C(s) G(s)}{1+C(s) G(s) H(s)}
$$


A função objetiva é definida, então, pela eq. (20):

$$
o=|m-1,1|
$$

Onde tem-se que:

$C(s)$ : Controlador PID

$G(s)$ : Planta a ser controlada

$H(s)$ : Ganho da retroalimentação

$Y(s)$ : Saída do sistema em malha fechada

$X(s)$ : Entrada do sistema em degrau unitário

$o$ : Função objetiva

$m$ : Máximo sobressinal

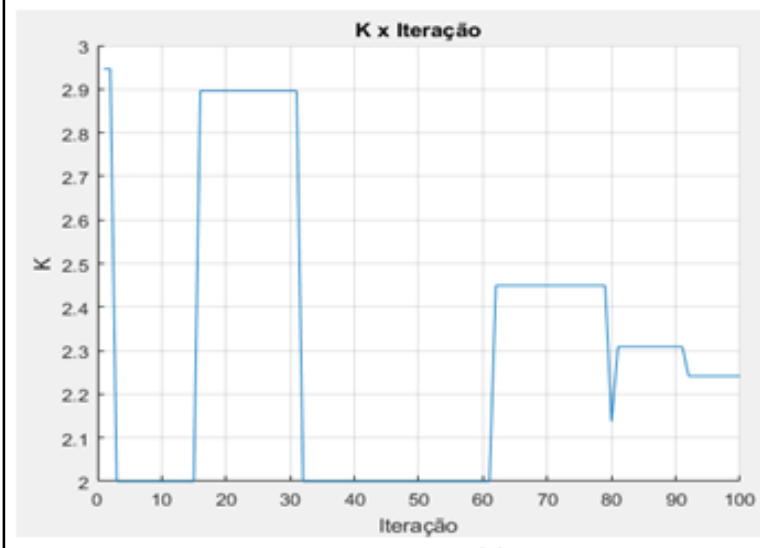

(a)

\section{RESULTADOS E DISCUSSÕES}

Como parâmetro para as simulações, foram adotados 40 agentes de busca (lobos) e 100 iterações. De cada iteração, é colhido o melhor valor encontrado para os parâmetros $\boldsymbol{K}$ e $\boldsymbol{a}$ do controlador com base no erro entre o valor encontrado e o máximo sobressinal de $10 \%$, definido pela eq. 3.7. Abaixo a figura 5 (a) e (b) representa as variações destes parâmetros a cada iteração.

Figura 5: Variação dos parâmetros do controlador x Iteração. (a) Parâmetro K. (b) Parâmetro $\boldsymbol{a}$.

Fonte: Autor, (2017).

O histórico de busca, representado na figura 6, apresenta a forma como os parâmetros se aproximam do melhor valor. Basicamente, indicam a trajetória dos lobos no encalço da presa, o princípio fundamental desta técnica de otimização. O Círculo vermelho indica o melhor resultado das iterações:

$$
\begin{gathered}
K=2.2415 \\
a=0.93302
\end{gathered}
$$

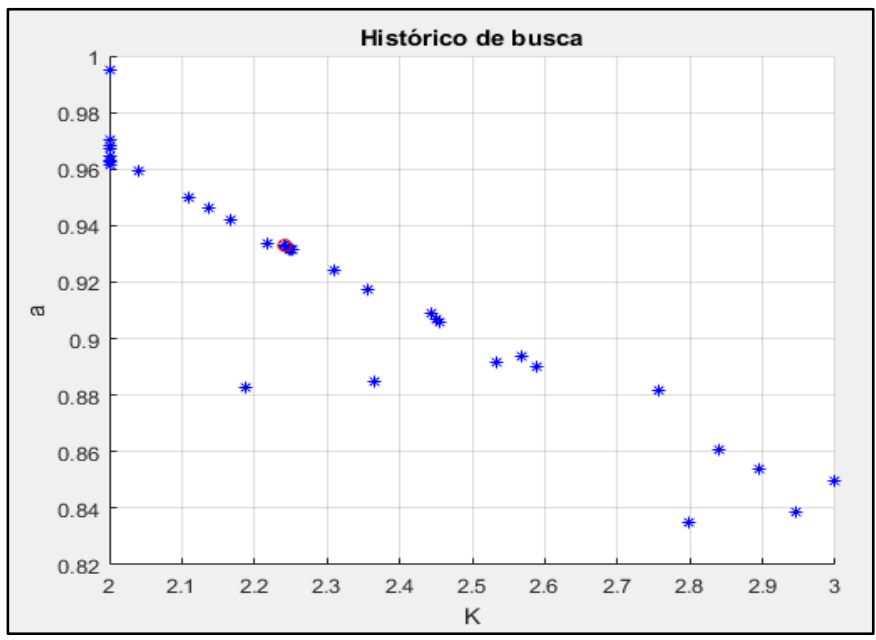

Figura 6: Histórico de busca.

Fonte: Autor, (2017)

A figura 7 apresenta o comportamento do erro a cada iteração, podendo ser verificada a diminuição rápida desde as primeiras cinco iterações. A partir de então, o algoritmo busca evitar os mínimos locais, com uma queda mais lenta até a última iteração.

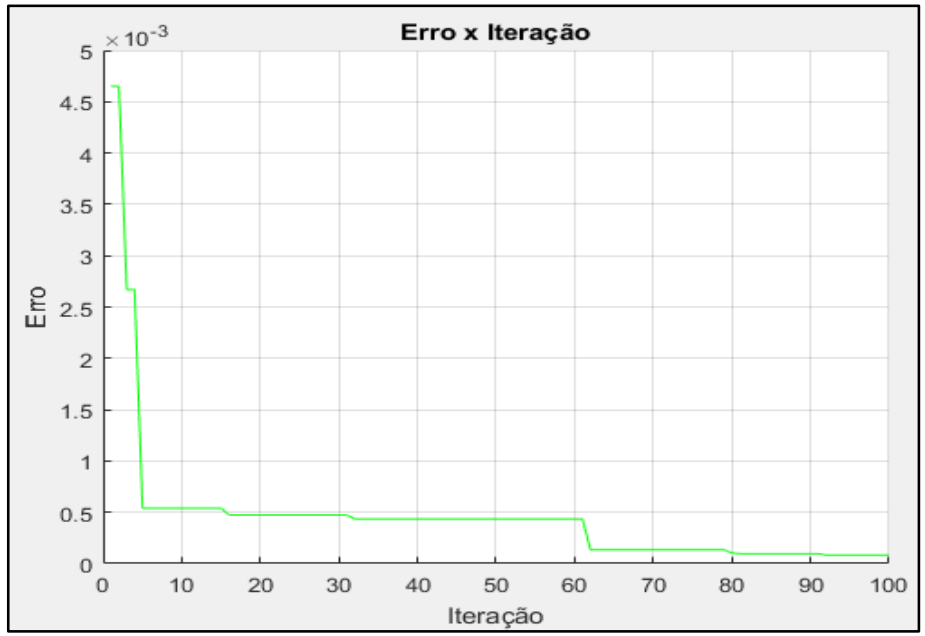

Figura 7: Erro x Iteração.

Fonte: Autor, (2017).

Para a primeira iteração, o quadro 1 apresenta os valores do sobressinal e do erro. É possível notar o caráter hierárquico da aptidão dos lobos, onde o erro aumenta partindo do $\alpha$ para os demais lobos.

Quadro 1: Parâmetro do controlador PID utilizando GWO.

\begin{tabular}{|l|l|l|l|l|}
\hline Lobo & $\boldsymbol{K}$ & $\boldsymbol{A}$ & Sobressinal & Erro \\
\hline $\boldsymbol{\alpha}$ & 2.915736 & 0.840386 & 1.0942 & 0.0058 \\
\hline $\boldsymbol{\beta}$ & 2.823458 & 0.849984 & 1.0934 & 0.0066 \\
\hline $\boldsymbol{\delta}$ & 2.034446 & 0.973289 & 1.1082 & 0.0082 \\
\hline
\end{tabular}

Fonte: Autor, (2017).

A resposta no domínio do tempo para o melhor valor encontrado segue na figura 8 . O valor do sobressinal máximo é obtido com uma diferença de $8.455 \mathrm{e}-05$. A forma de onda da 
resposta atende aos requisitos do problema, mantendo o sistema subamortecido e com sobressinal em $10 \%$ com o erro citado acima. O quadro 2 apresenta os dados resumidos.

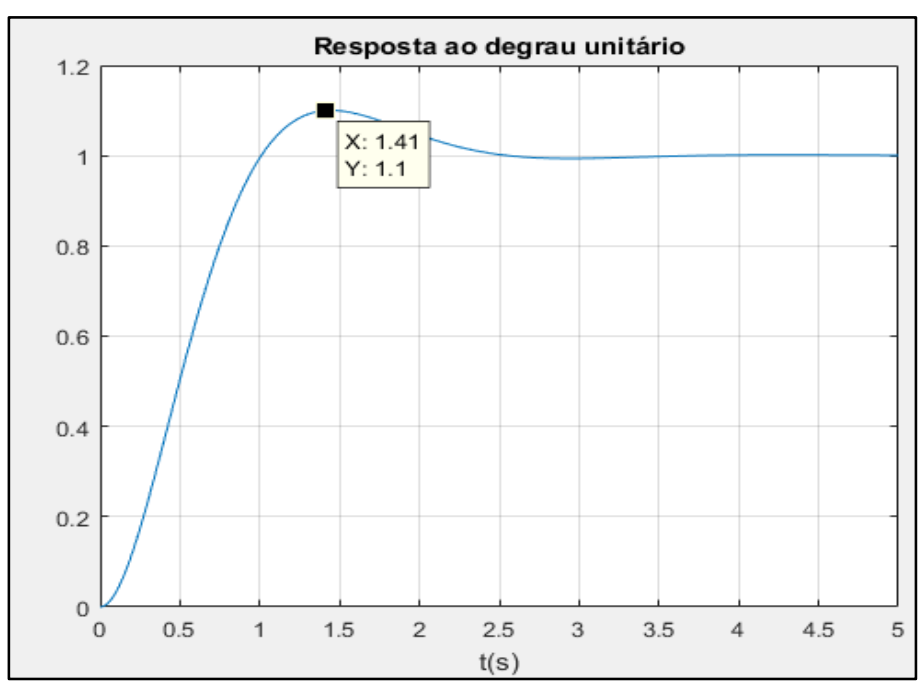

Figura 8: Resposta ao degrau unitário.

Fonte: Autor, (2017).

Quadro 2: Respostas do melhor valor obtido.

\begin{tabular}{|l|l|l|l|}
\hline $\boldsymbol{K}$ & $\boldsymbol{a}$ & Sobressinal & Erro \\
\hline 2.2415 & 0.93302 & 1.1001 & $8.14 \mathrm{E}-05$ \\
\hline
\end{tabular}

Fonte: Autor, (2017).

Nas figuras 9 e 10 seguem as respostas dos parâmetros de desempenho do algoritmo de otimização.

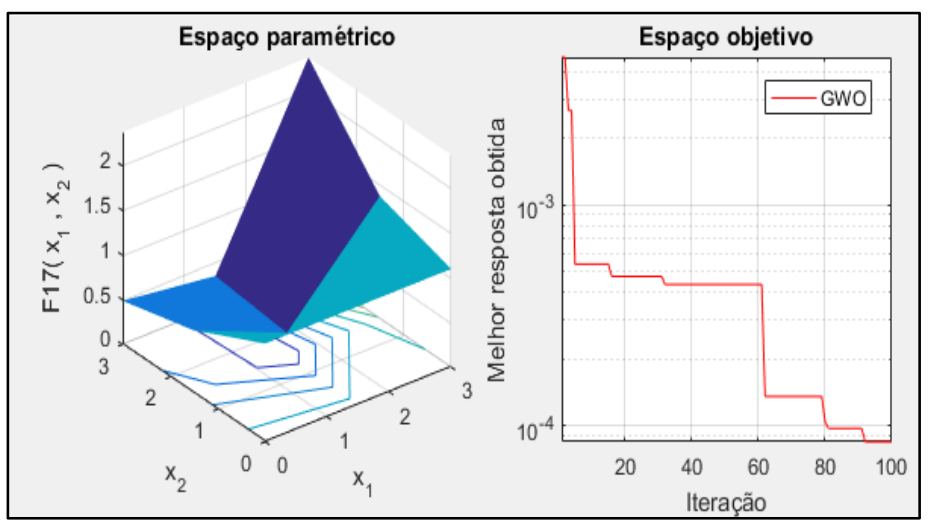

Figura 9: Curva de convergência.

Fonte: Autor, (2017).

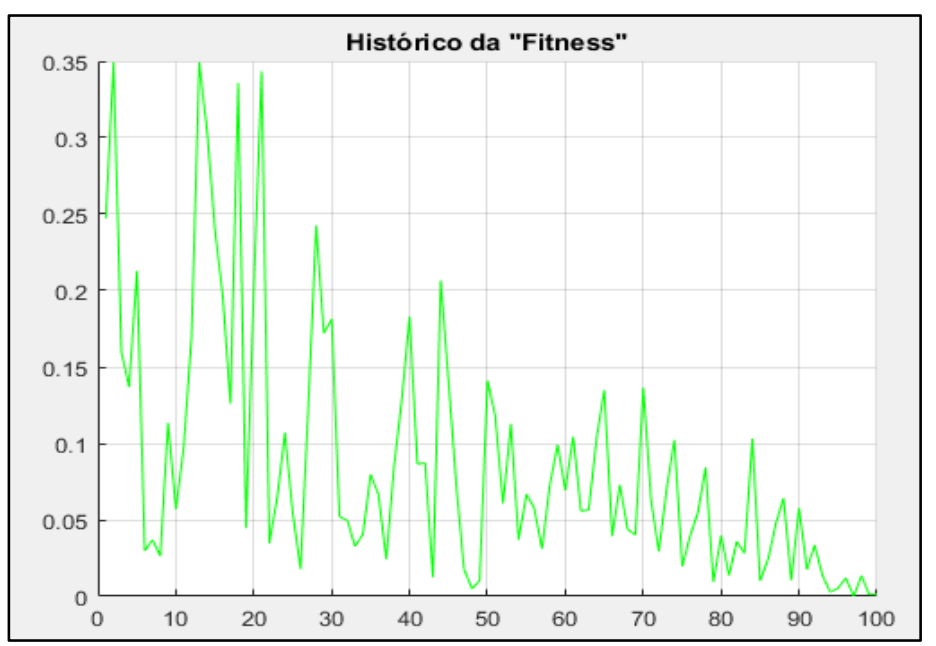

Figura 10:Histórico Fitness.

Fonte: Autor, (2017).
O método de tentativa e erro (T\&E) define os valores que serão testados de forma antecipada, não dando margem de escolha para otimização. Assim, para a resolução por este método, [1] definiu os seguintes valores de busca:]

$$
\begin{aligned}
& K=\left[\begin{array}{llllll}
2.0 & 2.2 & 2.4 & 2.6 & 2.8 & 3.0
\end{array}\right] \\
& a=\left[\begin{array}{llllll}
0.5 & 0.7 & 0.9 & 1.1 & 1.3 & 1.5
\end{array}\right]
\end{aligned}
$$

Para cada valor de $K$, todos os valores de $a$ são testados. O quadro 3 apresenta as respostas com esse método.

\begin{tabular}{|c|c|c|c|}
\hline $\mathrm{K}$ & $\mathrm{a}$ & Sobressinal & Erro \\
\hline 2 & 0.5 & 0.9001566 & 0.1998434 \\
\hline 2 & 0.7 & 0.9807257 & 0.1192743 \\
\hline 2 & 0.9 & 1.0614291 & 0.0385709 \\
\hline 2 & 1.1 & 1.179301 & 0.079301 \\
\hline 2 & 1.3 & 1.290349 & 0.190349 \\
\hline 2 & 1.5 & 1.3957544 & 0.2957544 \\
\hline 2.2 & 0.5 & 0.9114464 & 0.1885536 \\
\hline 2.2 & 0.7 & 0.9836907 & 0.1163093 \\
\hline 2.2 & 0.9 & 1.0771591 & 0.0228409 \\
\hline 2.2 & 1.1 & 1.1938201 & 0.0938201 \\
\hline 2.2 & 1.3 & 1.3038408 & 0.2038408 \\
\hline 2.2 & 1.5 & 1.4082899 & 0.3082899 \\
\hline 2.4 & 0.5 & 0.9206711 & 0.1793289 \\
\hline 2.4 & 0.7 & 0.9859183 & 0.1140817 \\
\hline 2.4 & 0.9 & 1.0923256 & 0.0076744 \\
\hline 2.4 & 1.1 & 1.2075283 & 0.1075283 \\
\hline 2.4 & 1.3 & 1.316343 & 0.216343 \\
\hline 2.4 & 1.5 & 1.4197563 & 0.3197563 \\
\hline 2.6 & 0.5 & 0.9283232 & 0.1716768 \\
\hline 2.6 & 0.7 & 0.9876528 & 0.1123472 \\
\hline 2.6 & 0.9 & 1.1068523 & 0.0068523 \\
\hline 2.6 & 1.1 & 1.2204687 & 0.1204687 \\
\hline 2.6 & 1.3 & 1.3279947 & 0.2279947 \\
\hline 2.6 & 1.5 & 1.4303577 & 0.3303577 \\
\hline 2.8 & 0.5 & 0.9347556 & 0.1652444 \\
\hline 2.8 & 0.7 & 1.0023519 & 0.0976481 \\
\hline 2.8 & 0.9 & 1.1207245 & 0.0207245 \\
\hline 2.8 & 1.1 & 1.232712 & 0.132712 \\
\hline 2.8 & 1.3 & 1.3389574 & 0.2389574 \\
\hline 2.8 & 1.5 & 1.4401856 & 0.3401856 \\
\hline 3 & 0.5 & 0.9402247 & 0.1597753 \\
\hline 3 & 0.7 & 1.017734 & 0.082266 \\
\hline 3 & 0.9 & 1.1339496 & 0.0339496 \\
\hline 3 & 1.1 & 1.2443132 & 0.1443132 \\
\hline 3 & 1.3 & 1.3492382 & 0.2492382 \\
\hline 3 & 1.5 & 1.4493184 & 0.3493184 \\
\hline
\end{tabular}

Quadro 3:Resposta com o método de tentativa e erro.

Fonte: Autor, (2017).

Foram realizadas 50 simulações para o cálculo do erro médio e do desvio padrão, comparando o desempenho do entre o GWO, PSO (Particle Swarm Optimization) e tentativa e erro (T\&E), apresentados no quadro 4.

Quadro 4 - Erro médio e desvio padrão.

\begin{tabular}{|l|l|l|l|}
\hline & GWO & PSO & T\&E \\
\hline Erro médio & $1.61 \mathrm{E}-01$ & $3.85 \mathrm{E}-03$ & 0.0149 \\
\hline Desvio padrão & $2.24 \mathrm{E}-01$ & $1.83 \mathrm{E}-02$ & 0.1798 \\
\hline
\end{tabular}

Fonte: Autor, (2017). 


\section{CONCLUSÃO}

Neste artigo, um controlador PID foi ajustado para que a saída do sistema atinja um sobressinal de $10 \%$. O método de otimização computacional GWO foi utilizado, tendo suas respostas comparadas com o PSO e o método de tentativa e erro (T\&E), este último adotado pelo autor do problema abordado. As comparações entre as técnicas foram validadas no MATLABß. Embora o esforço computacional seja maior, é possível notar uma diferença de até quatro casas decimais no valor do erro entre as técnicas que envolvem a tomada de decisão e a tentativa e erro. Em aplicações onde a precisão não seja um fator importante, os métodos menos eficientes podem ser adotados. Contudo, em sistemas onde o erro deve ser minimizado, compensa-se a utilização de técnicas meta heurísticas de otimização.

\section{AGRADECIMENTOS}

Ao Instituto de Tecnologia e Educação Galileo da Amazônia (ITEGAM), ao PPGEP/ITEC/UFAPA pelo apoio à pesquisa.

\section{REFERÊNCIAS BIBLIOGRÁFICAS}

[1] Ogata, K. Engenharia de controle moderno. 5.ed. São Paulo: Pearson Prentice Hall, 2010.

[2] Torres, W.L; Araujo, I. B. Q; Costa Junior, A.G. Mathematical Modeling and PID Controller Parameter Tuning in a Didactic Thermal Plant. IEEE Latin America Transactions. Vol. 15. 2017. p $1250-1256$.

[3] Mirjalili, S; Mohammad, S; Lewis, A. Grey Wolf Optimizer. Advances in Engineering Software. Elsevier. Ed. 69. 2014. p 4661 .

[4] Laskawski, M; Wcislik, M. New Optimal Settings Of PI and PID Controllers for The First-order Inertia and Dead Time Plant. 18th International Scientific Conference on Electric Power Engineering (EPE). 2017. p 1-6.

[5] Ibrahim, O; Yahaya, N. Comparative Studies of PID Controller Tuning Methods on a DC-DC Boost Converter. 6th International Conference on Intelligent and Advanced Systems (ICIAS). 2016.

[6] Long, W; Songjin, X. A novel grey wolf optimizer for global optimization. Advanced Information Management, Communicates, Electronic and Automation Control Conference (IMCEC), IEEE. 2016. p 1266-1270.

[7] Debnath, M; Jena, T; Mallick, R. Novel PD-PID Cascaded Controller for Automatic Generation Control of Amulti-area Interconnected Power System Optimized by Grey Wolf Optimization (GWO). 1st IEEE International Conference on Power Electronics, Intelligent Control and Energy Systems. 2016. p. 1-6.

[8] Yadav, S; Verma, S; Nagar, S. Reduction and Controller Design for Fractional Order spherical tank system using GWO. International Conference on Emerging Trends in Electrical, Electronics and Sustainable Energy Systems (ICETEESES-16). 2016. P 174-178. 